\title{
Yin-Yang: Concealing the Deep Embedding of DSLs
}

\author{
Vojin Jovanović ${ }^{\dagger}$ \\ Amir Shaikhha ${ }^{\dagger}$ \\ Christoph $\mathrm{Koch}^{\dagger}$
}

${ }^{\dagger}$ EPFL, Switzerland: \{firstname $\} .\{$ lastname $\}$ epfl.ch

\author{
Sandro Stucki ${ }^{\dagger}$ \\ Martin Odersky ${ }^{\dagger}$ \\ *University ITMO, Russia: \{firstname $\}.\{$ lastname $\} @$ cs.ifmo.ru
}

\begin{abstract}
Deeply embedded domain-specific languages (EDSLs) intrinsically compromise programmer experience for improved program performance. Shallow EDSLs complement them by trading program performance for good programmer experience. We present Yin-Yang, a framework for DSL embedding that uses Scala macros to reliably translate shallow EDSL programs to the corresponding deep EDSL programs. The translation allows program prototyping and development in the user friendly shallow embedding, while the corresponding deep embedding is used where performance is important. The reliability of the translation completely conceals the deep embedding from the user. For the DSL author, Yin-Yang automatically generates the deep DSL embeddings from their shallow counterparts by reusing the core translation. This obviates the need for code duplication and leads to reliability by construction.
\end{abstract}

Categories and Subject Descriptors $\quad$ D.3.3 [Programming Languages]: Language Constructs and Features

General Terms Languages, Design

Keywords Embedded Domain-Specific Languages, Deep Embedding, Shallow Embedding, Reflection, Macros

\section{Introduction}

Domain-specific languages (DSLs) provide a restricted, high-level, and user-friendly interface crafted for a specific domain. Restricting the language to a particular domain allows programming at a high level of abstraction while retaining good run-time performance by leveraging domain knowledge for optimized code generation or interpretation. In certain cases, code can even be targeted at heterogeneous computing environments [23].

The implementation of a usable external (or stand-alone) DSL requires building a parser, type-checker, and possibly a complete tool chain consisting of IDE integration, debugging, and documentation tools. This is a great undertaking that is often not justified by the benefits of having an external DSL. A promising alternative to external DSLs are embedded DSLs (EDSLs) [10] which are hosted in a general-purpose language and reuse its facilities. For the purpose of the following discussion, we distinguish between two main types of embeddings: shallow and deep embeddings.

Permission to make digital or hard copies of all or part of this work for personal or classroom use is granted without fee provided that copies are not made or distributed for profit or commercial advantage and that copies bear this notice and the full citation on the first page. Copyrights for components of this work owned by others than ACM must be honored. Abstracting with credit is permitted. To copy otherwise, or republish, to post on servers or to redistribute to lists, requires prior specific permission and/or a fee. Request permissions from Permissions@acm.org.

Copyright is held by the author/owner(s). Publication rights licensed to ACM.

GPCE'14, September 15-16, 2014, Västerås, Sweden

ACM 978-1-4503-3161-6/14/09

http://dx.doi.org/10.1145/2658761.2658771
- In a shallowly embedded DSL, values of the embedded language are directly represented by values in the host language. Consequently, terms in the host language that represent terms in the embedded language are evaluated directly into host-language values that represent DSL values. In other words, evaluation in the embedded language corresponds directly to evaluation in the host language.

- In a deeply embedded DSL, values of the embedded language are represented symbolically, that is, by host-language data structures, which we refer to as the intermediate representation $(I R)$. Terms in the host language that represent terms in the embedded language are evaluated into this intermediate representation. An additional evaluation step is necessary to reduce the intermediate representation to a direct representation. This additional evaluation is typically achieved through interpretation of the IR in the host language, or through code generation and subsequent execution.

An important advantage of deep embeddings over shallow ones is that DSL terms can be easily manipulated by the host language. This enables domain-specific optimizations $[20,23]$ that lead to orders-of-magnitude improvements in program performance, and multi-target code generation [2].

On the other hand, shallow embeddings typically suffer less from linguistic mismatch: this is particularly obvious for a class of shallow embeddings that we refer to as direct embeddings. Direct embeddings preserve the intrinsic constructs of the host language "on the nose". That is, DSL constructs such as if statements, loops, or function literals, as well as primitive data types such as integers, floating-point numbers, or strings are represented directly by the corresponding constructs of the host language.

Deep EDSLs intrinsically compromise programmer experience by leaking their implementation details (\$3.2). Often, IR construction is achieved through complex type system constructs that are, inevitably, visible in the EDSL interface. This can lead to cryptic type errors that are often incomprehensible to DSL users. In addition, the IR complicates program debugging as programmers cannot easily relate their programs to the code that is finally executed. Finally, the host language often provides more constructs than the embedded language and the usage of these constructs can be undesired in the DSL. If these constructs are generic in type (e.g., list comprehensions or try $\backslash$ catch) they can not be restricted in the embedded language by using complex types $(\$ 3.2)$.

Ideally, we would like to complement the high performance of deeply embedded DSLs, along with their capabilities for multi-target code generation, with the usability of their directly embedded counterparts. Reaching this goal turns out to be more challenging than one might expect: let us compare the interfaces of a direct embed- 
ding and a deep embedding of a simple EDSL for manipulating vectors ${ }^{1}$. The direct version of the interface is declared as:

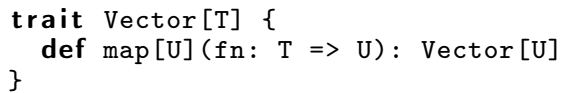

The interface of the deep embedding, however, fundamentally differs in the types: while the (polymorphic) map operation in the direct embedding operates directly on values of some generic type $\mathrm{T}$, the deep embedding must operate on whatever intermediate representations we chose for T. For our example, we chose the abstract, higher-kinded type Rep [T] to represent values of type T in the deep embedding:

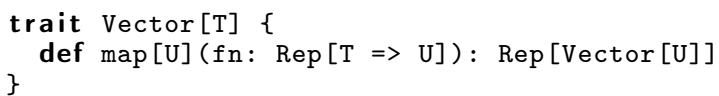

The difference in types is necessarily visible in the signature and thus inevitably leaks into user programs. This might seem like a low price to pay for all the advantages offered by a deep embedding. However, as we will see in $\S 3.2$, this difference in types is at the heart of many of the inconveniences associated with deep embeddings. How then, can we conceal this fundamental difference?

In Forge [29], Sujeeth et al. propose maintaining two parallel embeddings, shallow and deep, with a single interface equivalent to the deep embedding. In the shallow embedding, Rep is defined to be the identity on types, that is Rep [T] = T, effectively identifying IR types with their direct counterparts. As a result, shallowly embedded programs may be executed directly to allow for easy prototyping and debugging. In production, a simple "flip of a switch" enables the deep embedding. Unfortunately, artifacts of the deep embedding still leak to the user through the fundamentally "deeply typed" interface. We would like to preserve the idiomatic interface of the host language and completely conceal the deep embedding.

The central idea of this paper is the use of reflection to convert programs written in an unmodified direct embedding into their deeply embedded counterparts. Since the fundamental difference between the interfaces of the two embeddings resides in their types, we employ a configurable type translation to map directly embedded types $T$ to their deeply embedded counterparts $\llbracket T \rrbracket$. For our motivating example the type translation is simply:

$$
\begin{array}{lll}
\llbracket T \rrbracket=T & \text { if } T \text { is in type argument position, } \\
\llbracket T \rrbracket=\operatorname{Rep}[T] & \text { otherwise. }
\end{array}
$$

In $\$ 4$ we describe this translation among several others and discuss their trade-offs.

Together with a corresponding translation on terms, the type translation forms the core of Yin-Yang, a generic framework for DSL embedding, that uses Scala's macros [3] to reliably translate directly embedded DSL programs into corresponding deeply embedded DSL programs. The virtues of the direct embedding are used during program development when performance is not of importance; the translation is applied when performance is essential or alternative interpretations of a program are required (e.g., for hardware generation). To avoid error prone maintenance of synchronized direct and deep embeddings Yin-Yang reuses the core translation to generate the deep embeddings based on the definition of direct embeddings. Since the same translation is applied both for the EDSL definition and the EDSL program the equivalence between the embeddings is assured.

Yin-Yang contributes to the state of the art as follows:

- It completely conceals leaky abstractions of deep EDSLs from the users. The virtues of the direct embedding are used for pro-

\footnotetext{
1 All code examples are written in Scala. Similar techniques can be applied in other statically typed languages. Cf. [4, 14, 30].
}

totyping, while the deep embedding enables high-performance in production. The reliable translation ensures that programs written in the direct embedding will always be correct in the deep embedding. The core translation is described in $\$ 4$.

- It restricts host language features in the direct EDSL based on the supported features of the deep EDSL. Specialized type checking of the translated direct EDSL displays comprehensible errormessages to the user. Language restriction is further described in $\$ 5$.

- It simplifies deep EDSL development and guarantees semantic equivalence between the direct embedding and the deep embedding by reusing the core translation to generate the deep EDSL definition out of the direct EDSL definition $(\S 6)$.

We evaluate Yin-Yang by generating 3 deep EDSLs from their direct embedding, and providing interfaces for 2 existing EDSLs. The effects of concealing the deep embedding and reliability of the translation were evaluated on 21 programs (1284 LOC), from EDSLs OptiGraph [27] and OptiML [28]. In all programs combined the direct implementation obviates 101 type annotations related to the deep embedding. The complete evaluation is presented in $\$ 7$.

Throughout the paper, we target the LMS [20] framework as a deep embedding back-end due to the plethora of existing LMS EDSLs. Consequently, we will assume that deep embeddings use LMS' extensible IR. However, Yin-Yang is applicable to other types of IR (e.g., polymorphic embeddings [9]) and possibly other statically typed languages $(§ 8)$.

\section{Background on Scala}

In this section we provide background information necessary for understanding Yin-Yang's implementation in Scala. We briefly explain the core concepts of Lightweight Modular Staging [20, 23] and Scala Macros [3]. Throughout the paper we assume familiarity with the basics of the Scala Programming Language [17].

\subsection{Deep Embedding of DSLs with LMS}

Lightweight Modular Staging (LMS) is a staging [31] framework and an embedded compiler for developing deeply embedded DSLs. LMS provides a library of reusable language components organized as traits (Scala's first-class modules). An EDSL developer selects traits containing the desired language features, combines them through mix-in composition [16] and adds DSL-specific functionality to the resulting EDSL trait. EDSL programs then extend this trait, inheriting the selected LMS and EDSL language constructs. Figure 1 illustrates this principle. The trait VectorDSL defines a simplified EDSL for creating and manipulating vectors over some numeric type T. Two LMS traits are mixed into the VectorDSL trait: the Base trait introduces core LMS constructs and the NumericOps trait introduces the Numeric type class and the corresponding support for numeric operations. The bottom of the figure shows an example usage of the EDSL. The constant literals in the program are lifted to the IR through implicit conversions introduced by NumericOps [18].

All types in the VectorDSL interface are instances of the parametric type Rep [_]. The Rep [_] type is an abstract type member of the Base LMS trait and abstracts over the concrete types of the IR nodes that represent DSL operations in the deep embedding. Its type parameter captures the type underlying the IR: EDSL terms of type Rep [T] evaluate to host language terms of type T during EDSL execution.

Operations on Rep [T] terms are added by implicit conversions that are introduced in the EDSL scope. For example, the implicit class VectorOps introduces the + operation on every term of 


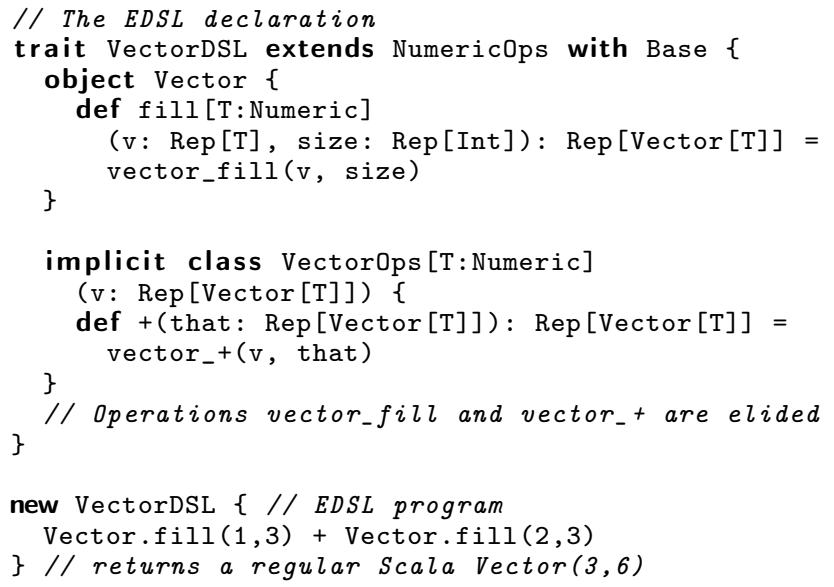

Figure 1: Minimal EDSL for vector manipulation.

type Rep [Vector [T] ]. In the example, the type class Numeric ensures that vectors contain only numerical values.

LMS has been successfully used by in project Delite $[2,27]$ for building DSLs that support heterogeneous parallel computing. EDSLs developed with Delite cover domains such as machine learning, graph processing, data mining, etc. Due to its wide use and high performance we choose Delite as a back-end for Yin-Yang.

\subsection{Scala Macros}

Scala Macros [3] are a compile-time meta-programming feature of Scala. Macros operate on Scala abstract syntax trees (ASTs): they can construct new ASTs, or transform and analyze the existing Scala ASTs. Macro programs can use common functionality of the Scala compiler like error-reporting, type checking, transformations, traversals, and implicit search.

Yin-Yang uses a particular flavor of Scala macros called def macros, though we will often drop the prefix "def" for the sake of brevity. From a programmer's point of view, def macros are invoked just like regular Scala methods. However, macro invocations are expanded during compile time to produce new ASTs. Macro invocations are type checked both before and after expansion to ensure that expansion preserves well-typedness. Macros have separated declarations and definitions: declarations are represented to the user as regular methods while macro definitions operate on Scala ASTs. The arguments of macro method definitions are the type-checked ASTs of the macro arguments.

For DSLs based on Yin-Yang we use a macro that accepts a single block of code as its input. At compile time, this block is first type checked against the interface of the direct embedding. Then, Yin-Yang applies the generic transformation to translate the directly embedded AST to the corresponding deeply embedded AST. For example, given the following DSL snippet, Yin-Yang produces the VectorDSL program in Figure 1:

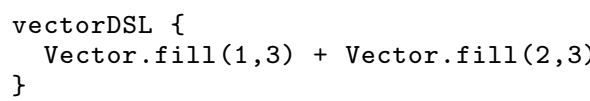

\section{Motivation}

The main idea of this paper is that EDSL users should program in a direct embedding, while the corresponding deep embedding should be used only in production. To motivate this idea we consider the direct embedding and the deep embedding of a simple EDSL for manipulating vectors. Here, we use Scala to show the problems with the deep embedding that apply to other statically typed programming

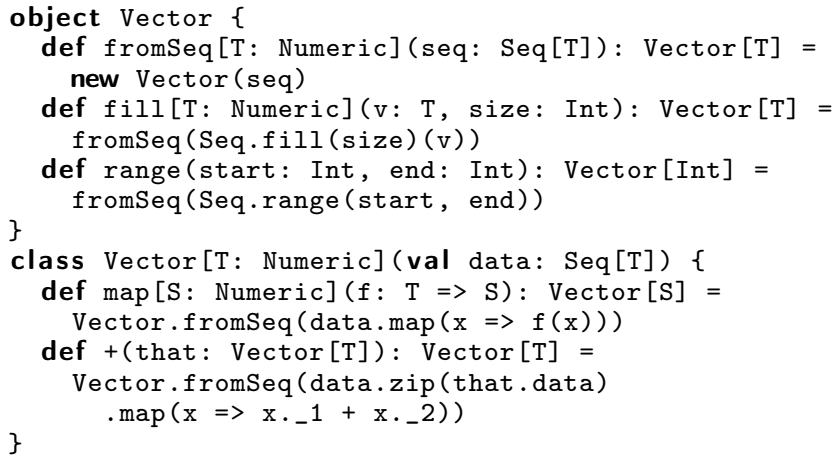

Figure 2: The interface of a direct EDSL for manipulating numerical vectors.

languages (e.g., Haskell and $\mathrm{OCaml}$ ). These languages achieve the embedding in different ways $[4,8,14,30]$, but this is always reflected in the type signatures. In the context of Scala, there are additional problems with type inference and implicit conversions, however, we omit those from the discussion as language specific.

Figure 2 shows a simple direct EDSL for manipulating numerical vectors. Vectors are instances of a Vector class, and have only two operations: $i$ ) vector addition (the + ), and $i i$ ) the higher-order map function which applies a function $f$ to each element of the vector. The Vector object provides factory methods fromSeq, range, and $f i l l$ for vector construction. Note that though the type of the elements in a vector is generic, we require it to be an instance of the Numeric type class.

For a programmer, this is an easy to use library. Not only can we write expressions such as $\mathrm{v} 1+\mathrm{v} 2$ for summing vectors (resembling mathematical notation), but we can also get meaningful type error messages. The EDSL is an idiomatic Scala and displayed type errors are comprehensible. Finally, in the direct embedding, all terms directly represent values from the embedded language and inspecting intermediate values with the debugger is straightforward.

The problem, however, is that the code written in such a direct embedding suffers from major performance issues [23]. For some intuition, consider the following code for adding 3 vectors: $\mathrm{v} 1+\mathrm{v} 2+\mathrm{v} 3$. Here, each + operation creates an intermediate Vector instance, uses the zip function, which itself creates an intermediate Seq instance, and calls a higher-order map function. The abstractions of the language that allow us to write code with high-level of abstraction have a downfall in terms of performance. Consecutive vector summations would perform much better if they were implemented with a simple while loop.

\subsection{The Deep Embedding}

For the DSL from Figure 2, the overhead could be eliminated with optimizations like stream fusion [5] and inlining, but to properly exploit domain knowledge, and to potentially target other platforms, one must introduce an intermediate representation of the EDSL program. The intermediate representation can be transformed according to the domain-specific rules (e.g., eliminating addition with a null vector) to improve performance beyond common compiler optimizations [23]. To this effect, we use the LMS framework and present the deep version of the EDSL for manipulating numerical vectors in Figure 3.

In the VectorDSL interface every method has an additional implicit parameter of type SourceContext and every generic type requires an additional TypeTag type class. The SourceContext contains information about the current file name, line number, and character offset. SourceContexts are used for mapping generated 


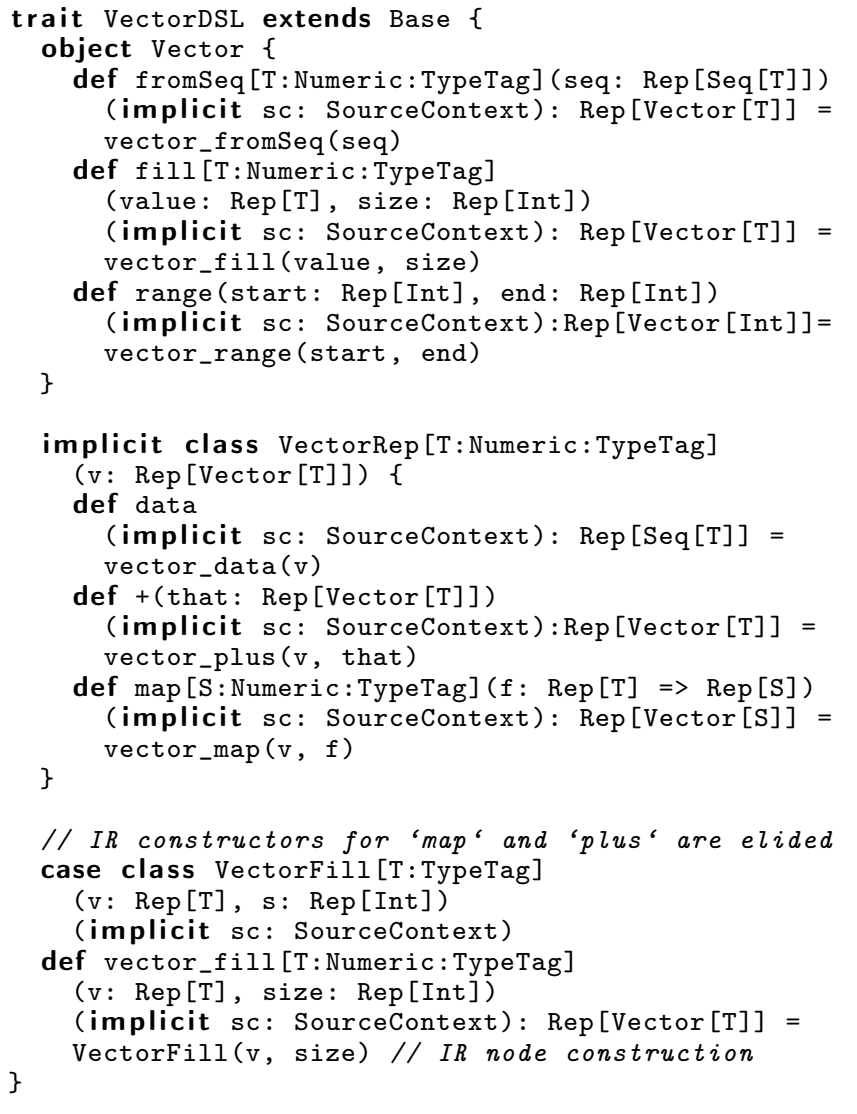

Figure 3: A LMS based deep EDSL for manipulating numerical vectors.

code to the original program source. TypeTags carry all information about the type of terms. They are used to propagate run-time type information through the EDSL compilation for optimizations and generating code for statically typed target languages. In the EDSL definitions the SourceContext is rarely used explicitly (i.e., as an argument). It is provided "behind the scenes" by implicit definitions that are provided in the DSL.

\subsection{Abstraction Leaks in the Deep Embedding}

The programs in the deep embedding construct the IR instead of the values in the embedded language. This inevitably leaks to the users in the following ways:

Convoluted interfaces. The interface of the EDSL has Rep [_] types in all its method signatures. Furthermore, once we introduce code generation, the method signatures must be enriched with source and type information (SourceContext and TypeTag) and inevitably become complex. This makes the interface very complicated to understand. The user of the EDSL, who might not be an expert programmer, needs to understand concepts like TypeTag and SourceContext to grasp the interface.

Difficult debugging. In the methods of the direct EDSL all terms directly represent values in the embedded language (there is no intermediate representation). This allows users to trivially use debugging tools to step through the terms and inspect the values of the embedded language. With the deep EDSL, method definitions only instantiate the IR nodes. In the classical debugging mode this does not convey any useful information to the user. Furthermore, debugging generated code or an interpreter is extremely difficult.
Users cannot relate the debugger position and the original line of code.

Complicated Type Errors. The Rep [_] types leak to the user through type errors. Even for simple type errors the user is exposed to non-standard error messages. In certain cases (e.g., incorrect call to an overloaded function), the error messages can become hard to understand. To illustrate, we present a typical type error for invalid method invocation:

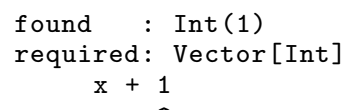

In the deep embedding the corresponding type error contains Rep types and the this qualifier:

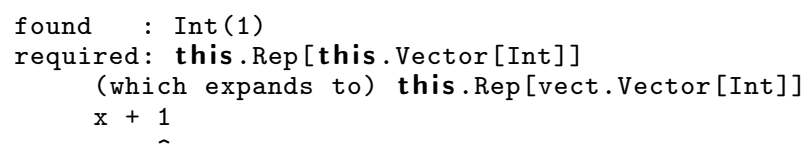

This example represents one of the most common type errors. For more complicated type errors cf. [13].

Unrestricted host language constructs. In the deep embedding all generic constructs of a host language can be used arbitrarily. For example, scala.List.fill[T] (count: Int, el: T) can, for the argument el, accept both direct and deep terms. This is often undesirable as it can lead to code explosion and unexpected program behavior.

In the following example, assume that generic methods $f i l l$ and reduce are not masked by the VectorDSL and belong only to the host language library. In this case, the invocation of $f i l l$ and reduce performs meta-programming over the IR of the deep embedding:

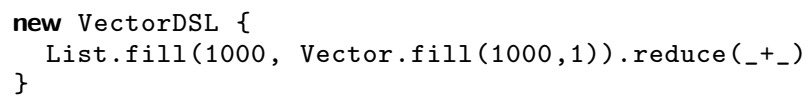

Here, at DSL compilation time, the program creates a Scala list that contains a thousand IR nodes for the Vector.fill operation and performs a vector addition over them. Instead of producing a small IR the compilation result is a thousand IR nodes for vector addition. This is a typical case of code explosion that could not happen in the direct embedding which does not introduce an IR.

On the other hand, some operations can be completely ignored. In the next example, the try/catch block will be executed during EDSL compilation instead during DSL program execution:

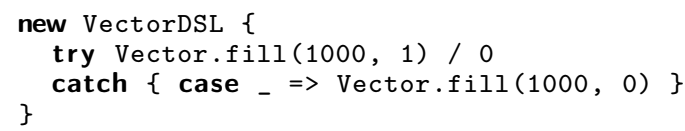

Here, the resulting program always throws a DivisionByZero exception.

\section{Translation of the Direct Embedding}

The purpose of the core Yin-Yang translation is to reliably and automatically make a transition from a directly embedded DSL program to its deeply embedded counterpart. The transition requires a translation for the following reasons: $i$ ) host language constructs such as if statements are strongly typed and accept only primitive types for some of their arguments (e.g., a condition has to be of type Boolean), ii) all types in the direct embedding need to be translated into their IR counterparts (e.g., Int to Rep [Int]), iii) the directly embedded DSL operations need to be mapped onto their deeply embedded counterparts, and $i v$ ) methods defined in the deep embedding require additional parameters, such as run-time type 


$$
\begin{gathered}
\Gamma \vdash t: T_{2} \\
\frac{\Gamma x: T_{1} \Rightarrow t \rrbracket=\operatorname{lam}\left[T_{1}, T_{2}\right]\left(x: T_{1} \Rightarrow \llbracket t \rrbracket\right)}{\Gamma \vdash t_{1} \cdot f:\left[T_{1}\right]\left(T_{2} \Rightarrow T_{3}\right)} \\
\frac{\operatorname{\llbracket t} t_{1} \cdot f\left[T_{1}\right]\left(t_{2}\right) \rrbracket=\operatorname{app}\left[T_{2}, T_{3}\right]\left(\llbracket t_{1} \rrbracket \cdot f\left[T_{1}\right], \llbracket t_{2} \rrbracket\right)}{\llbracket \operatorname{def} f\left[T_{1}\right]\left(x: T_{2}\right): T_{3}=t \rrbracket=\operatorname{def} f\left[T_{1}\right]:\left(T_{2} \Rightarrow T_{3}\right)=\llbracket x: T_{2} \Rightarrow t \rrbracket} \\
\llbracket \operatorname{val} x: T=t \rrbracket=\operatorname{val} x: T=\operatorname{valDef}[T](t) \\
\frac{\Gamma \vdash \operatorname{if}\left(t_{1}\right) t_{2} \text { else } t_{3}: T}{\llbracket \text { if }\left(t_{1}\right) t_{2} \text { else } t_{3} \rrbracket=\text { ifThenElse }[T]\left(\llbracket t_{1} \rrbracket, \llbracket t_{2} \rrbracket, \llbracket t_{3} \rrbracket\right)}
\end{gathered}
$$

Figure 5: Subset of rules for language virtualization.

information and source positions. To address these inconsistencies we propose a straightforward solution: a type-directed program translation from direct to deep embeddings.

Since the translation is type-directed it requires reflection that supports type introspection and type transformation. The translation is based on the idea of representing language constructs as method calls $[4,22]$ and systematically intrinsifying direct DSL operations and types of the direct embedding to their deep counterparts [4]. The translation operates in two main steps:

Language virtualization converts host language intrinsics into function calls, which can then be evaluated to the appropriate IR values in the deep embedding.

EDSL intrinsification converts DSL intrinsics (operations and types) from the direct embedding into their deep counterparts.

To illustrate the core translation, we use an example program for calculating $\sum_{i=0}^{n} i^{e x p}$ using the vector EDSL defined in Figure 2. Figure 4 contains three versions of the program: Figure $4 \mathrm{a}$ depicts the direct embedding version, Figure $4 \mathrm{~b}$ represents the program after type checking (as the translation sees it), and Figure 4c shows the result of the translation.

Language virtualization allows to redefine intrinsic constructs of the host language, such as if and while statements. This can be achieved by translating them into suitable method invocations as shown by Rompf et al. in the modified Scala compiler named Scala-Virtualized [22].

Yin-Yang follows the same approach as Scala-Virtualized but uses macros of unmodified Scala to virtualize Scala intrinsics required to write direct DSL programs. In addition to Scala-Virtualized we virtualize function definition and application, and variable binding. Furthermore, Scala is designed such that the types Any and AnyRef, which reside at the top of the Scala class hierarchy, contain final methods; through inheritance, these methods are defined on all types making it impossible to override their functionality. Since Yin-Yang uses unmodified Scala we must virtualize all methods on Any and AnyRef.

Figure 4 illustrates this process and Figure 5 lists a subset of the translation rules used to virtualize the Scala intrinsics, with $\llbracket t \rrbracket$ denoting the translation of a term $t$. Note that method definitions ${ }^{2}$ need to be translated into function definitions in order to be virtualized. In all expressions the original types are introspected and used as a type argument of the virtualized method. These generic types are translated later during the DSL intrinsification phase in order to avoid type inference in future stages of the translation.

\footnotetext{
${ }^{2}$ In Scala, the def keyword is used to define (possibly recursive) methods. This is similar to the let and letrec constructs in other functional languages.
}

Constructs that are not virtualized are class and trait definitions, including case class definitions, and pattern matching. We are planning to add the latter in future versions of Yin-Yang.

DSL intrinsification maps directly embedded versions of the DSL intrinsics to their deep counterparts. The constructs that need to be converted are: $i$ ) DSL types, ii) DSL operations, iii) constant literals, and $i v$ ) captured variables in the direct program:

- The type translation maps every DSL type in the, already virtualized, term body to an equivalent type in the deep embedding. In other words, the type translation is a function on types. Note that this function is inherently DSL-specific, and hence needs to be configurable by the DSL author. We discuss aspects of different type translation in more detail in $\$ 4.1$.

The type mapping depends on the input type and the context. In practice, we need only distinguish between types in typeargument position, e.g. the type argument Int in the polymorphic function call lam [Int, Int], and the others. To this end, we define a pair of mutually recursive functions $\tau_{\text {arg }}, \tau: \mathbb{T} \rightarrow \mathbb{T}$ where $\mathbb{T}$ is the set of all types and $\tau_{\text {arg }}$ and $\tau$ translate types in argument and non-argument positions, respectively.

- The operation translation maps directly embedded versions of the DSL operations into corresponding deep embeddings. To this end, we define a function opMap on terms that returns deep operation for each directly embedded operation. For deep embeddings based on LMS, or polymorphic embeddings [9] in general, opMap simply injects operations into the scope of the deep EDSL (i.e., by adding the prefix this). Of course, other approaches, such as name mangling or importing definitions from a different module, are also possible. In the current implementation of Yin-Yang, the opMap function is fixed to simply inject the this prefix, although this might change in the future. In Figure 4, calls to range on the object vector. Vector and pow on the package object math . 'package ' are respectively translated to calls range and pow on this. Vector and this. 'package'. For simplicity, passing source information (SourceContext) and type information TypeTag is handled implicitly by the Scala compiler. In absence of implicit parameters they should be handled by the translation.

- Constants can be intrinsified in the deep embedding in multiple ways. They can be converted to a method call for each constant (e.g., $\llbracket 1 \rrbracket=\ldots 1$ ), type (e.g., $\llbracket 1 \rrbracket=\operatorname{liftInt~(1)),~or~with~a~}$ unified polymorphic function (e.g., $\llbracket 1 \rrbracket=\operatorname{lift}[\operatorname{Int}]$ (1)). In the example, we use the polymorphic function approach for the constants.

- Free variables are external variables captured by a direct EDSL term. All that deep embedding knows about these terms is their type and that they will become available only during evaluation (i.e., interpretation or execution after code generation). Hence, free variables need to be treated specially by the translation and the deep embedding needs to provide support for their evaluation. In Figure 4, the free variables $n$ and exp are replaced with calls to the polymorphic method hole [T], which handles the evaluation of free variables in the deep embedding. Each captured identifier is assigned with a unique number that is, together with type information, passed as an argument to the hole method (0 and 1 in Figure 4). The identifiers are later sorted and passed as arguments to the Scala function that is a result of EDSL compilation. The DSL author is required to ensure that the position and the type of the resulting function matches the order and types of the sorted identifiers passed by Yin-Yang. 


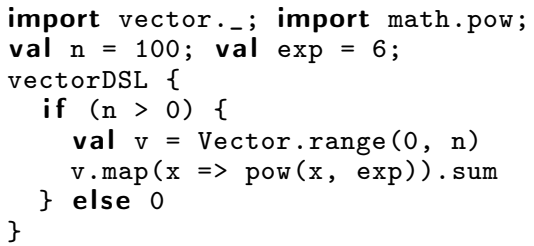

(a) A program in direct embedding for calculating $\sum_{i=0}^{n} i^{e x p}$. val $\mathrm{n}=100 ;$ val $\exp =6$; vectorDSL \{

if $(n>0)\{$

val $\mathrm{v}$ : Vector [Int] = vector. Vector.range $(0, n)$

$\mathrm{v} \cdot \operatorname{map}[$ Int] $(\mathrm{x}:$ Int $=>$ math. 'package'. pow (x, exp)

). sum [Int] (math. Numeric.IntIsIntegral)

\} else 0

(b) The original program after desugaring and type inference.

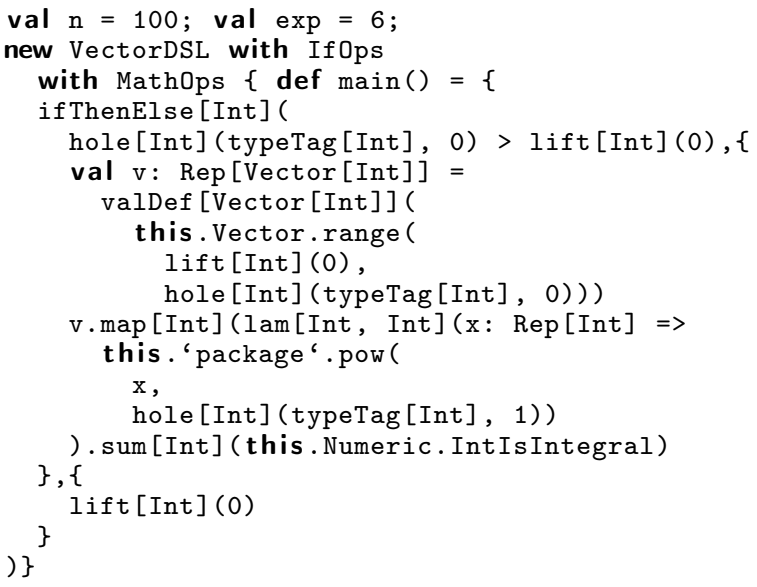

(c) The Yin-Yang translation of the program from Figure $4 \mathrm{~b}$.

Figure 4: Transformation of an EDSL program for calculating $\sum_{i=0}^{n} i^{e x p}$.

\subsection{Alternative Type Translations}

Having type translation as a function opens a number of possible deep embedding strategies. Alternative type translations can also dictate the interface of lam and app and other core EDSL constructs. Here we discuss the ones that we find useful in EDSL design:

The identity translation. If we choose $\tau$ to be the identity function and virtualization methods such as lam, app and if ThenElse to be implemented in the obvious way using the corresponding Scala intrinsics, the resulting translation will simply yield the original, directly embedded DSL program.

Generic polymorphic embedding. If instead we choose $\tau$ to map any type term $T$ (in non-argument position) to Rep [T], for some abstract, higher-kinded IR type Rep in the deep EDSL scope, we obtain a translation to a finally-tagless, polymorphic embedding [4, 9]. For this embedding, the translation functions are defined as:

$$
\begin{aligned}
& \tau_{\arg }(T)=T \\
& \tau(T) \quad=\operatorname{Rep}[T]
\end{aligned}
$$

By choosing the virtualized methods to operate on the IR-types in the appropriate way, one obtains an embedding that preserves well-typedness, irrespective of the particular DSL it implements. We will not present the details of this translation here, but refer the interested reader to [4]

Eager inlining. In high-performance EDSLs it is often desired to eagerly inline all functions and to completely prevent dynamic dispatch in user code (e.g., storing functions into lists). This is achieved by translating function types of the form $A=>B$ in the direct embedding into $\operatorname{Rep}[A] \Rightarrow \operatorname{Rep}[B]$ in the deep embedding (where Rep again designates IR types). Instead of constructing an IR node for function application, such functions reify the whole body of the function starting with IR nodes passed as arguments. The effect of such reification is equivalent to inlining. This function representa- tion is used in LMS [20] by default and we use it in Figure 4. The translation functions are defined as:

$$
\begin{array}{ll}
\tau_{\arg }\left(T\left[I_{1}, \ldots, I_{n}\right]\right) & =T\left[\tau_{\arg }\left(I_{1}\right), \ldots, \tau_{\arg }\left(I_{n}\right)\right] \\
\tau_{\arg }\left(T_{1} \Rightarrow T_{2}\right) & =\text { error } \\
\tau_{\arg }(T) & =T, \text { otherwise } \\
\tau\left(T_{1} \Rightarrow T_{2}\right) & =\operatorname{Rep}\left[\tau_{\arg }\left(T_{1}\right)\right] \Rightarrow \operatorname{Rep}\left[\tau_{\arg }\left(T_{2}\right)\right] \\
\tau(T) & =\operatorname{Rep}[T], \text { otherwise }
\end{array}
$$

This translation preserves well-typedness but rejects programs that contain function types in the type-argument position. In this case this is a desired behavior as it fosters high-performance code by avoiding dynamic dispatch. As an alternative to rejecting function types in the type-argument position the deep embedding can provide coercions from $\operatorname{Rep}[A] \Rightarrow \operatorname{Rep}[B]$ to $\operatorname{Rep}[A=>B]$ and from $\operatorname{Rep}[A=>B]$ to $\operatorname{Rep}[A] \Rightarrow \operatorname{Rep}[B]$.

Untyped backend. If DSL authors want to avoid complicated types in the back-end (e.g., Rep [T] ), the $\tau$ functions can simply transform all types to the Dynamic [1] type. Giving away type safety can make transformations in the back-end easier for the DSL author.

Custom types. All previous translations preserved types in the type parameter position. The reason is that the $\tau$ functions behaved like a higher-kinded type. If we would like to map some of the base types in a custom way, those types need to be changed in the position of type-arguments as well. This translation is used for EDSLs based on polymorphic embedding [9] that use this . T to represent type T.

With the previous translations the type system of the direct embedding was ensuring that the term will type-check in the deep embedding. We applied this translation to Slick [32] with great success $(\$ 7.3)$.

Interestingly, just by changing the type translation, the EDSL author can modify the behavior of an EDSL. For example, with the generic polymorphic embedding the EDSL will reify function IR nodes and thus allow for dynamic dispatch. In the same EDSL that uses the eager inlining translation, dynamic dispatch is restricted and all function calls are inlined. 


\subsection{Correctness}

To completely conceal the deep embedding all type errors must be captured in the direct embedding or by the translation, i.e., the translation must never produce an ill-typed program. Proving this property is verbose and partially covered by previous work Therefore, for each version of the type translation we provide references to the previous work and give a high-level intuition:

- The identity translation ensures that well-typed programs remain well typed after the translation to the deep embedding [4]. Here the deep embedding is the direct embedding with virtualized host language intrinsics.

- Generic polymorphic embedding preserves well-typedness [4]. Type $\mathrm{T}$ is uniformly translated to Rep [T] and thus every term will conform to its expected type.

- Eager inlining preserves well-typedness for programs that are not explicitly rejected by the translation. We discuss correctness of eager inlining in [13] on a Hindley-Milner based calculus similar to the one of Carette et al. [4].

For the intuition why type arguments can not contain function types consider passing an increment function to the generic identity function:

$\operatorname{id}[\mathrm{T} \Rightarrow \mathrm{T}](\operatorname{lam}[\mathrm{T}, \mathrm{T}](\mathrm{x}=>\mathrm{x}+1))$

Here, the id function expects Rep [_] type but the argument is $\operatorname{Rep}[\mathrm{T}]=>\operatorname{Rep}[\mathrm{T}]$

- The Dynamic type supports all operations and, thus, static type errors will not occur. Here, the DSL author is responsible for providing a back-end where dynamic type errors will not occur.

- Custom types can cause custom type errors since EDSL authors can arbitrarily redefine types (e.g., type Int $=$ String. YinYang provides no guarantees for this type of the translation.

\section{Restricting Host Language Constructs}

The direct DSL programs can contain well-typed expressions that are not supported by the deep embedding. Often, these expressions lead to unexpected program behavior (\$3) and we must rule them out by reporting meaningful and precise error messages to the user.

We could rule out unsupported programs by relying on properties of the core translation. If a direct program contains unsupported expressions, after translation it will become ill-typed in the deep embedding. We could reject unsupported programs by simply reporting type checking errors. Since, the direct program is welltyped and the translation preserves well-typedness all type errors must be due to unsupported expressions.

Unfortunately, naively restricting the language by detecting typechecking failures is leaking information about the deep embedding The reported error messages will contain virtualized language constructs and types. This is not desirable as users should not be exposed to the internals of the deep embedding.

Yin-Yang avoids leakage of the deep embedding internals in error messages by performing an additional verification step that, in a fine grained way, checks if a method from the direct program exists in the deep embedding. This step traverses the tree generated by the core translation and verifies for each method call if it correctly typechecks in the deep embedding. If the type checking fails Yin-Yang reports two kinds of error messages:

- Generic messages for unsupported methods:

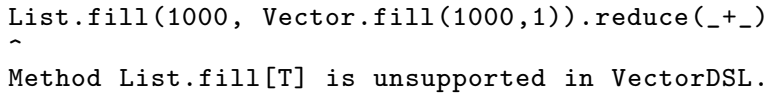

- Custom messages for unsupported host language constructs: try Vector.fill (1000, 1) / 0

Construct try/catch is unsupported in VectorDSL

With Yin-Yang the DSL author can arbitrarily restrict virtualized constructs in an embedded language by simply omitting corresponding method definitions from the deep embedding. Due to the additional verification step all error messages are clearly shown to the user. This allows easy construction of embedded DSLs that support only a subset of the host language.

\section{Automatic Generation of the Deep Embedding}

So far, we have seen how Yin-Yang translates programs written in the direct embedding to the deep embedding. This arguably simplifies life for EDSL users by allowing them to work with the interface of the direct embedding. However, the EDSL author still needs to maintain synchronized implementations of the two embeddings, which can be a tedious and error prone task.

To alleviate this issue, Yin-Yang automatically generates the deep embedding from the implementation of the direct embedding. This happens in two steps: First, we generate high-level IR nodes and methods that construct them through a systematic conversion of methods declared in a direct embedding to their corresponding methods in the deep embedding (\$6.1). Second, we exploit the fact that method implementations in the direct embedding are also direct DSL programs. Reusing our core translation from $\$ 4$, we translate them to their deep counterparts $(\S 6.2)$. In the translated method bodies, in addition to the translated DSL itself, we also allow usage of the Scala library constructs that supported by the target back-end (cf. [13]).

The automatic generation of deep embeddings reduces the amount of boilerplate code that has to be written and maintained by EDSL authors, allowing them to instead focus on tasks that can not be easily automated, such as the implementation of domainspecific optimizations in the deep embedding. However, automatic code generation is not a silver bullet. Hand-written optimizations acting on the IR typically depend on the structure of the later, introducing hidden dependencies between such optimizations and the direct embedding. Care must be taken in order to avoid breaking optimizations when changing the direct embedding of the EDSL.

For further information on how to use Yin-Yang's code generation together with the core translation, and how to specify rewrite rules, cf. [13].

\subsection{Constructing High-Level IR Nodes}

To make the generation regular Yin-Yang provides a corresponding IR node and construction method for every operation in the direct embedding. By using reflection, we extract the method signatures from the direct embedding. From these, we generate the interface, implementation, and code generation traits as prescribed by LMS. This part of the translation is LMS specific and applying it to other frameworks would require changing the code templates. Based on the signature of each method, we generate the case class that represents the IR node. Then, for each method we generate a corresponding method that instantiates the high-level IR nodes. Whenever a method is invoked in the deep EDSL, instead of being evaluated, a high-level IR node is created.

Figure 6 illustrates the way of defining IR nodes for Vector EDSL. The case classes in the VectorOps trait define the IR nodes for each method in the direct embedding. The fields of these case classes are the callee object of the corresponding method (e.g., $\mathrm{v}$ in VectorMap), and the arguments of that method (e.g., $f$ in VectorMap). 


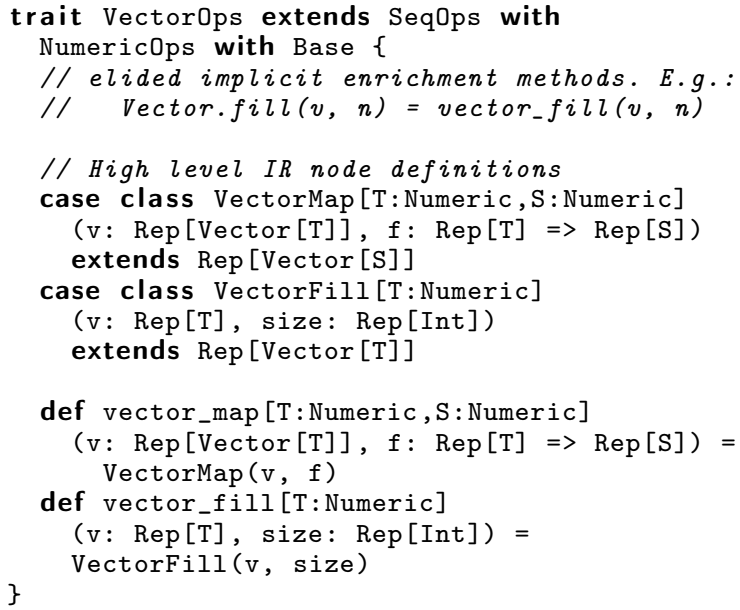

Figure 6: High-level IR nodes for Vector.

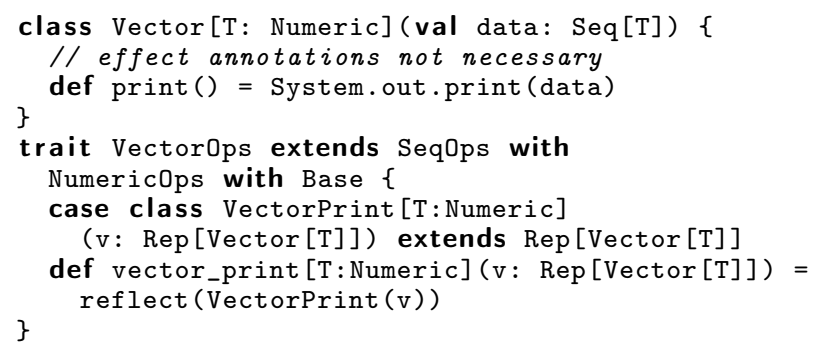

Figure 7: Direct and deep embedding for Vector with sideeffects.

Deep embedding should, in certain cases, be aware of sideeffects. The EDSL author must annotate methods that cause sideeffects with an appropriate annotation. To minimize the number of needed annotations we use Scala FX [25]. Scala FX is a compiler plugin that adds an effect system on top of the Scala type system. With Scala FX the regular Scala type inference also infers the effects of expressions. As a result, if the direct EDSL is using libraries which are already annotated, like the Scala collection library, then the EDSL author does not have to annotate the direct EDSL. Otherwise, there is a need for manual annotation of the direct embedding by the EDSL author. Finally, the Scala FX annotations are mapped to the corresponding effect construct in LMS.

Figure 7 shows how we automatically transform the I/O effect of a print method to the appropriate construct in LMS. As the Scala FX plugin knows the effect of System. out.println, the effect for the print method is inferred together with its result type (Unit). Based on the fact that the print method has an I/O effect, we wrap the high-level IR node creation method into reflect, which is an effect construct in LMS to specify an I/O effect [21]. In effect, all optimizations in the EDSL will have to preserve the order of println and other I/O effects. We omit details about the LMS effect system; for more details cf. [21].

\subsection{Lowering High-Level IR Nodes to their Low-Level Implementation}

Having domain-specific optimizations on the high-level representation is not enough for generating high performance code. In order to improve the performance, we must transform these high-level nodes into their corresponding low-level implementations. Hence,

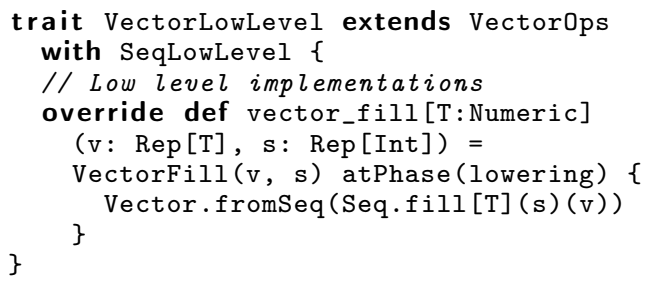

Figure 8: Lowering to the low-level implementation for Vector.

we must represent the low-level implementation of each method in the deep EDSL. After creating the high-level IR nodes and applying domain-specific optimizations, we transform these IR nodes into their corresponding low-level implementation. This can be achieved by using a lowering phase [23].

Figure 8 illustrates how the invocation of each method results in creating an IR node together with a lowering specification for transforming it into its low-level implementation. For example, whenever the method $\mathrm{fill}$ is invoked, a VectorFill IR node is created like before. However, this high-level IR node needs to be transformed to its low-level IR nodes in the lowering phase. This delayed transformation is specified using an atPhase (lowering) block [23]. Furthermore, the low-level implementation uses constructs requiring deep embedding of other interfaces. In particular, an implementation of the fill method requires the Seq.fill method that is provided by the SeqLowLevel trait.

Generating the low-level implementation is achieved by transforming the implementation of each direct embedding method. This is done in two steps. First, the expression given as the implementation of a method is converted to a Scala AST of the deep embedding by core translation of Yin-Yang. Second, the code represented by the Scala AST must be injected back to the corresponding trait. To this effect, we implemented Sprinter [15], a library that generates correct and human readable code out of Scala ASTs. The generated source code is used to represent the lowering specification of every IR node.

\section{Evaluation}

We compared the deep embedding generation of Yin-Yang with Forge on three Delite-based deep EDSLs: OptiML, OptiQL, and Vector (\$7.1). Then, we measured the effect of concealing the deep embedding by counting the number of obviated annotations related to deep embedding in the test suites of OptiML and OptiGraph EDSLs (§7.2). Finally, we evaluated the ease of adopting Yin-Yang for the existing deep EDSL Slick [32] (\$7.3) and compare the effort of designing the interface with the current version of the interface. We do not report on execution speed since performance benefits of the deep embedding have been studied previously [23, 29].

\subsection{Automatic Deep EDSL Generation}

To evaluate the automatic deep EDSL generation for OptiML, OptiQL, and Vector, we used Forge [29], a Scala based metaEDSL for generating both direct and deep EDSLs from a single specification. Forge already contained specifications for OptiML and OptiQL.

To avoid re-typing OptiML and OptiQL we modified Forge to generate the direct embedding from its specification and generated the direct embeddings from the existing Forge based EDSL specifications. Then, we used our automatic deep generation tool to convert these direct embeddings into their deep counterparts. Since, deep EDSLs mostly consist of boilerplate the generated embeddings have a similar number of LOC as the handwritten counterparts. For all 
three EDSLs, we verified that tests running in the direct embeddings behave the same as the tests for the deep embeddings.

In Table 1, we give a line count comparison for the code in the direct embedding, Forge specification, and deep embedding for three EDSLs: i) OptiML is a Delite-based EDSL for machine learning, ii) $O p t i Q L$ is a Delite-based EDSL for running in-memory queries, and iii) Vector is the EDSL shown as an example throughout this paper We are careful with measuring lines-of-code (LOC) with Forge and the deep EDSLs: we only count the parts which are generated out of the given direct EDSL.

Overall, Yin-Yang requires roughly the same number of LOC as Forge to specify the DSL. This can be viewed as positive result since Forge relies on a specific meta-language for defining the two embeddings. Yin-Yang, however, uses Scala itself for this purpose and is thus much easier to use. In case of OptiML, Forge slightly outperforms Yin-Yang. This is because Forge supports metaprogramming at the level of classes while Scala does not.

Table 1: LOC for direct EDSL, Forge specification, and deep EDSL.

\begin{tabular}{llll}
\hline EDSL & Direct & Forge & Deep \\
\hline OptiML & 1128 & 1090 & 5876 \\
OptiQL & 73 & 74 & 526 \\
Vector & 70 & 71 & 369 \\
\hline
\end{tabular}

We did not compare the efforts required to specify the DSL with Yin-Yang and Forge. The reason is twofold:

- It is hard to estimate the effort required to design a DSL. If the same person designs a single DSL twice, the second implementation will always be easier and take less time. On the other hand, when multiple people implement a DSL their skill levels can greatly differ. Finally, DSL design is technically demanding and it is hard to find a large enough group to conduct a statistically significant user study.

- Writing the direct embedding in Scala is arguably simpler than writing a Forge specification. Forge is Delite-specific language and uses a custom preprocessor to define method bodies in Scala Thus, learning a new language and combining it with Scala snippets must be harder than just writing idiomatic Scala.

\subsection{No Annotations in the Direct Embedding}

To evaluate the number of obviated annotations related to the deep embedding we implemented a direct embedding for the OptiGraph EDSL (an EDSL for graph processing), and used the generated direct EDSL for OptiML. We implemented the whole application suites of these EDSLs with the direct embedding. All 21 applications combined have 1284 lines of code.

To see the effects of the direct embedding as the front-end we counted the number of deep embedding related annotations that were used in the application suite. The counted annotations are Rep [T] for types and lift $(t)$ for lifting literals when implicit conversions fail. In 21 applications the direct embedding obviated 96 Rep [T] annotations and 5 lift $(t)$ annotations.

\subsection{Yin-Yang for Slick}

Slick is a deeply embedded Scala EDSL for database querying and access. Slick is not based on LMS, but still uses Rep types to achieve reification. To improve the complicated interface of Slick we used Yin-Yang. However, since the deep embedding of Slick already exists, we first designed the new interface (direct embedding). The new interface has dummy method implementations since semantics of different database back-ends can not be mapped to Scala. Thus, this interface is used only for user friendly error reporting and documentation. The interface is completely new, covers all the functionality of Slick, and consists of only 70 lines of code (cf. [13]).

Slick has complicated method signatures that do not correspond to the simple new interface. In order to preserve backward compatibility, the redesign of Slick to fit Yin-Yang's core translation was not possible. We addressed this by adding a wrapper for the deep embedding of Slick that fits the required signature. The wrapper contains only 240 lines of straightforward code.

We compare the effort required for the interface design with Yin-Yang and with traditional type system based approaches. The development of the previous Slick interface required more than a year of development while the Yin-Yang version was developed in less than one month. The new front-end passes all 54 tests that cover the most important functionalities of Slick. When using Slick all error messages are idiomatic to Scala and resemble typical error messages from the standard library.

This study was performed by only two users and, thus, is not statistically significant. Still, we find the difference in required effort large enough to indicate that Yin-Yang simplifies front-end development of EDSLs.

\section{Discussion}

Yin-Yang consistently translates terms to the embedded domain and, thus, postpones DSL compilation to run-time. Although, compilation happens in a different compilation stage, Yin-Yang does not allow staging [31]. EDSLs can, however, achieve partial evaluation [12] if their implementation supports it.

We implemented Yin-Yang in Scala, however the underlying principles are applicable in the wider context. Yin-Yang operates in the domain of statically typed languages based on the HindleyMilner calculus with a type system that is advanced enough to support deep EDSL embedding. The type inference mechanism, purity, laziness, and sub-typing, do not affect the operation of YinYang. Different aspects of Yin-Yang require different language features, which we discuss separately below.

The core translation and language restriction are based on term and type transformations. Thus, the host language must support reflection, introspection and transformation on types and terms. This can be achieved both at run-time and compile-time.

Semantic equivalence between the direct embedding and deep embedding is required for debugging and prototyping. If there is a semantic mismatch [6] between the two embeddings, e.g., the host language is lazy and the embedded language is strict, Yin-Yang can not be used for debugging. In this scenario the direct embedding can be implemented as stub which is used only for its user friendly interface and error reporting.

\section{Related Work}

Yin-Yang is a framework for developing embedded DSLs in the spirit of Hudak [10, 11]: embedded DSLs are Scala libraries and DSL programs are just Scala programs that do not, in general, require preor post-processing using external tools. Yin-Yang translates directly embedded DSL programs into finally-tagless [4] deep embeddings. Our approach supports (but is not limited to) polymorphic [9] deep embeddings, and - as should be apparent from the examples used in this paper - is particularly well-adapted for deep EDSLs using an LMS-type IR [22, 23].

As discussed in $\S 1$, Sujeeth et al. propose Forge [29], a Scala based meta-EDSL for generating equivalent shallow and deep embeddings from a single specification. DSLs generated by Forge provide a common abstract interface for both shallow and deep 
embeddings through the use of abstract Rep types. A shallow embedding is obtained by defining Rep as the identity function on types, i.e. $\operatorname{Rep}[\mathrm{T}]=\mathrm{T}$.

A DSL user can switch between the shallow and deep embeddings by changing a single flag in the project build. Unfortunately, the interface of the shallow embedding generated by Forge remains cluttered with Rep type annotations. Additionally, some plain types that are admissible in a directly embedded program may lack counterparts among the IR types of the deep embedding. This means that some seemingly well-typed DSL programs become ill-typed once the transition from the shallow to the deep embedding is made, forcing users to manually fix type errors in the deeply embedded program. Finally, DSL authors must learn a new language for EDSL design whereas with Yin-Yang this language is Scala itself.

Project Lancet [24] by Rompf et al. and work of Scherr and Chiba [26] interpret Java bytecode to extract domain-specific knowledge from directly embedded DSL programs compiled to bytecode. These solutions are similar to Yin-Yang in that the direct embedding is translated to the deep embedding, however, they do not provide functionality to generate a deep embedding out of a direct one.

\section{Conclusions}

We presented Yin-Yang, a framework for DSL embedding that completely conceals the unfriendly interface of the deep embedding, while still leveraging all their benefits. We believe Yin-Yang is an important step towards wider adoption of embedded DSLs. If Yin-Yang would be used in combination with custom type-error reporting of Scalad [19] and syntax extensibility of frameworks like SugarJ [7], embedded DSLs would provide most benefits of the external DSLs, while, due to automatic generation of the deep embedding, DSL implementation is significantly simplified.

\section{Acknowledgments}

We thank all the reviewers of this work for their valuable feedback and suggestions. This research was sponsored by the European Research Council (ERC) under grant 587327 "DOPPLER".

\section{References}

[1] M. Abadi, L. Cardelli, B. Pierce, and G. Plotkin. Dynamic typing in a statically typed language. ACM Transactions on Programming Languages and Systems (TOPLAS), 13(2):237-268, 1991

[2] K. J. Brown, A. K. Sujeeth, H. J. Lee, T. Rompf, H. Chafi, M. Odersky, and K. Olukotun. A heterogeneous parallel framework for domainspecific languages. In Parallel Architectures and Compilation Techniques (PACT), 2011.

[3] E. Burmako. Scala macros: Let our powers combine! In Workshop on Scala (SCALA), 2013

[4] J. Carette, O. Kiselyov, and C.-c. Shan. Finally tagless, partially evaluated: Tagless staged interpreters for simpler typed languages Journal of Functional Programming, 19(05):509-543, 2009.

[5] D. Coutts, R. Leshchinskiy, and D. Stewart. Stream fusion: From lists to streams to nothing at all. In International Conference on Functional Programming (ICFP), 2007.

[6] K. Czarnecki, J. O'Donnell, J. Striegnitz, and W. Taha. DSL implementation in MetaOCaml, Template Haskell, and C++. Domain-Specific Program Generation, page 51-72, 2004

[7] S. Erdweg, T. Rendel, C. Kästner, and K. Ostermann. Sugarj: Librarybased syntactic language extensibility. In Object-Oriented Programming Systems, Languages, and Applications (OOPSLA), 2011.

[8] M. Guerrero, E. Pizzi, R. Rosenbaum, K. Swadi, and W. Taha. Implementing DSLs in metaOCaml. In Object-Oriented Programming Systems, Languages, and Applications (OOPSLA), 2004.

[9] C. Hofer, K. Ostermann, T. Rendel, and A. Moors. Polymorphic embedding of DSLs. In International Conference on Generative Programming and Component Engineering (GPCE), 2008.
[10] P. Hudak. Building domain-specific embedded languages. $A C M$ Computing Surveys, 28(4es):196, 1996.

[11] P. Hudak. Modular domain specific languages and tools. In International Conference on Software Reuse (ICSR), 1998.

[12] N. D. Jones, C. K. Gomard, and P. Sestoft. Partial Evaluation and Automatic Program Generation. Prentice-Hall, Inc., 1993.

[13] V. Jovanovic, A. Shaikhha, S. Stucki, V. NIkolaev, C. Koch, and M. Odersky. Yin-Yang: Concealing the deep embedding of DSLs. Technical Report EPFL-REPORT-200500, EPFL Lausanne, Switzerland, 2014.

[14] T. Lokhorst. Awesome prelude, 2012. Dutch Haskell User Group, http://vimeo.com/9351844.

[15] V. Nikolaev. Sprinter. http://vladimirnik.github.io/ sprinter/.

[16] M. Odersky and M. Zenger. Scalable component abstractions. In Object-Oriented Programming Systems, Languages, and Applications (OOPSLA), 2005.

[17] M. Odersky, P. Altherr, V. Cremet, B. Emir, S. Micheloud, N. Mihaylov, M. Schinz, E. Stenman, and M. Zenger. The Scala Language Specification. 2011.

[18] B. C. Oliveira, A. Moors, and M. Odersky. Type classes as objects and implicits. In Object-Oriented Programming Systems, Languages, and Applications (OOPSLA), 2010.

[19] H. Plociniczak. Scalad: an interactive type-level debugger. In Workshop on Scala (SCALA), 2013.

[20] T. Rompf and M. Odersky. Lightweight modular staging: a pragmatic approach to runtime code generation and compiled DSLs. Communications of the ACM, 55(6):121-130, June 2012.

[21] T. Rompf, A. Sujeeth, H. Lee, K. Brown, H. Chafi, M. Odersky, and K. Olukotun. Building-blocks for performance oriented DSLs. In IFIP Working Conference on Domain-Specific Languages (DSL), 2011.

[22] T. Rompf, N. Amin, A. Moors, P. Haller, and M. Odersky. Scalavirtualized: Linguistic reuse for deep embeddings. Higher-Order and Symbolic Computation, page 1-43, 2013.

[23] T. Rompf, A. K. Sujeeth, N. Amin, K. J. Brown, V. Jovanović, H. Lee, M. Jonnalagedda, K. Olukotun, and M. Odersky. Optimizing data structures in high-level programs: New directions for extensible compilers based on staging. In Symposium on Principles of Programming Languages (POPL), 2013.

[24] T. Rompf, A. K. Sujeeth, K. J. Brown, H. Lee, H. Chafi, K. Olukotun, and M. Odersky. Project Lancet: Surgical precision JIT compilers. In International Conference on Programming Language Design and Implementation (PLDI), 2013.

[25] L. Rytz, M. Odersky, and P. Haller. Lightweight polymorphic effects. In European Conference on Object-Oriented Programming (ECOOP), 2012.

[26] M. Scherr and S. Chiba. Implicit staging of EDSL expressions: A bridge between shallow and deep embedding. In European Conference on Object-Oriented Programming (ECOOP), 2014.

[27] A. Sujeeth, T. Rompf, K. Brown, H. Lee, H. Chafi, V. Popic, M. Wu, A. Prokopec, V. Jovanović, M. Odersky, and K. Olukotun. Composition and reuse with compiled domain-specific languages. In European Conference on Object-Oriented Programming (ECOOP), 2013.

[28] A. K. Sujeeth, H. Lee, K. J. Brown, T. Rompf, H. Chafi, M. Wu, A. Atreya, M. Odersky, and K. Olukotun. OptiML: An implicitly parallel domain-specific language for machine learning. In International Conference on Machine Learning (ICML), 2011.

[29] A. K. Sujeeth, A. Gibbons, K. J. Brown, H. Lee, T. Rompf, M. Odersky, and K. Olukotun. Forge: generating a high performance DSL implementation from a declarative specification. In International Conference on Generative Programming and Component Engineering (GPCE), 2013.

[30] J. Svenningsson and E. Axelsson. Combining deep and shallow embedding for EDSL. In Trends in Functional Programming (TFP), pages 21-36, 2013.

[31] W. Taha and T. Sheard. Multi-stage programming with explicit annotations. In Workshop on Partial Evaluation and Program Manipulation (PEPM), 1997

[32] Typesafe. Slick. http://slick.typesafe.com/. 